\title{
Digital Filter Design: Global Solutions via Polynomial Optimization
}

\author{
Wu-Sheng Lu \\ Dept. of Electrical and Computer Engineering \\ University of Victoria \\ Victoria, BC, Canada V8W 3P6 \\ Email: wslu@ece.uvic.ca
}

\begin{abstract}
This paper aims at introducing a new design tool based on polynomial optimization, which can be used to obtain globally optimal designs for stable IIR filters, single-stage and multistage frequency response masking filters, and digital filters with discrete coefficients, and many other types of filters and filter banks. We show how these design problems can be formulated as polynomial optimization problems (POPs) and present a simple numerical example to demonstrate the usefulness of this new design framework.
\end{abstract}

\section{INTRODUCTION}

Designing digital filters for various signal processing applications has been a subject of study for more than three decades and, as a result, many effective design methods have been documented [1]-[3]. On the other hand, this field of research continues to be in full of vigor as new design problems arise and innovative design techniques emerge. Roughly speaking, there are three kinds of design problems where finding global solutions presents significant challenges. The first kind of problems are IIR type designs that are intrinsically nonlinear and nonconvex. The second kind of problems are FIR type designs with certain structures that lead to nonconvex second-order or higher-than-2nd-order designs. An example in this class is the design of FIR frequency-response masking (FRM) filters [4]. In the third kind of problems, one is concerned with digital filters with discrete coefficients. An example in this class is the design of FIR (or IIR) filters with sum of power of two (SP2) coefficients [5][6].

In this paper, we introduce a new design framework based on the recently proposed semidefinite programming (SDP) relaxation algorithms for polynomial optimization problems (POPs) [10][13]. The new framework is illustrated by applying it to three design problems mentioned above. A simple numerical example concerning a 2nd-order stable IIR filters is presented to introduce some software for POPs and demonstrate the usefulness of the new framework.

\section{Polynomial Optimization Problems}

\section{A. Polynomial Optimization Problems}

A real-valued polynomial $f(\boldsymbol{x})$ in $n$-dimensional space $R^{n}$ can be expressed as

$$
f(\boldsymbol{x})=\sum_{\boldsymbol{\alpha} \in \mathcal{F}} c(\boldsymbol{\alpha}) \boldsymbol{x}^{\boldsymbol{\alpha}}
$$

where $c(\boldsymbol{\alpha}) \in R$ and $\boldsymbol{x}^{\boldsymbol{\alpha}}=x_{1}^{\alpha_{1}} x_{2}^{\alpha_{2}} \ldots x_{n}^{\alpha_{n}}$ for every $\boldsymbol{x} \in R^{n}$ and every $\boldsymbol{\alpha}=\left[\begin{array}{llll}\alpha_{1} & \alpha_{2} & \ldots & \alpha_{n}\end{array}\right] \in \mathcal{F} \subset \mathcal{Z}_{+}^{n}$ with $\mathcal{Z}_{+}^{n}$ the set of all vectors in $R^{n}$ whose components are nonnegative integers. The order (degree) $m$ of $f(\boldsymbol{x})$ is defined as the largest $\sum_{i} \alpha_{i}$ amongst all nonzero $c(\boldsymbol{\alpha})$. A general POP can be expressed as

$$
\begin{aligned}
\operatorname{minimize} & f_{0}(\boldsymbol{x}) \\
\text { subject to: } & f_{k}(\boldsymbol{x}) \geq 0 \quad \text { for } k=1, \ldots, K
\end{aligned}
$$

where $f_{k}(\boldsymbol{x})$ for $k=0,1, \ldots, K$ are real-valued polynomials. Obviously, POPs include linear programming (LP), convex quadratic programming (QP), semidefinite programming (SDP), and second-order cone programming (SOCP) problems as its special cases. However as we shall illustrate, POPs stand for a substantially broader class that covers many important optimization problems which do not belong to any of the above-mentioned convex programming sub-classes.

\section{B. Global Optimization of Problem (2)}

Studies on global optimization with polynomials have started two decades ago [7] and have since made important progress along the way [8][9]. A recent breakthrough in the field is made by Lasserre [10] in which it is proved that when the feasible region defined by (2b) is compact (not necessarily convex), the global solution of (2) can be approximated as closely as desired (and often can be obtained exactly) by solving a finite sequence of SDP problems. A problem with the method of [10] is that the sizes of the SDP problems involved in a POP usually grows very quickly that may cause numerical difficulties even for POPs of moderate scales. Very recently, sparse SDP relaxation [11] is proposed for computing global solution of POPs of larger scales with improved efficiency.

In the rest of the paper, we illustrate the usefulness of the POP framework by applying it to three distinct filter design problems.

\section{DESIGN OF StABlE IIR FILTERS}

Consider the design of a stable IIR digital filter whose transfer function

$$
H(z)=\frac{\sum_{i=0}^{n} a_{i} z^{i}}{\prod_{i=1}^{k}\left(b_{i 0}+b_{i 1}+z^{2}\right)}
$$

is supposed to approximate a desired frequency response $H_{d}(\omega)=H_{d r}(\omega)-j H_{d i}(\omega)$ in $L_{\infty}$-norm. In (3), for the sake of simplicity we have assumed an even $n$ and $k=n / 2$, but 
with straightforward modifications the formulation given below is immediately appliable to the case with an odd $n$. By placing an upper bound for $W(\omega)\left|H(\omega)-H_{d}(\omega)\right|^{2}$ on $\Omega \subset[-\pi, \pi]$ where $W(\omega)$ is a known weighting function and $\Omega$ denotes the frequency region of interest, the design problem at hand can be formulated as

$$
\begin{aligned}
\operatorname{minimize} & \delta \\
\text { subject to: } & W(\omega)\left|H(\omega)-H_{d}(\omega)\right|^{2} \leq \delta \quad \omega \in \Omega \\
& H(z) \text { is stable }
\end{aligned}
$$

Let $\boldsymbol{a}=\left[\begin{array}{lll}a_{0} & \ldots & a_{n}\end{array}\right]^{T}, \boldsymbol{b}=\left[\begin{array}{lllll}b_{10} & b_{11} & \ldots & b_{k 0} & b_{k 1}\end{array}\right], \boldsymbol{c}(\omega)=$ $\left[\begin{array}{llll}1 & \cos \omega & \ldots & \cos n \omega\end{array}\right]^{T}$, and $s(\omega)=\left[\begin{array}{llll}0 & \sin \omega & \ldots & \sin n \omega\end{array}\right]^{T}$, the frequency response of the IIR filter is given by

$$
\begin{aligned}
= & \frac{\boldsymbol{a}^{T} \boldsymbol{c}(\omega)+j \boldsymbol{a}^{T} \boldsymbol{s}(\omega)}{\prod_{i=1}^{k}\left[\left(b_{i 0}+b_{i 1} \cos \omega+\cos 2 \omega\right)+j\left(b_{i 1} \sin \omega+\sin 2 \omega\right)\right]} \\
= & \frac{\boldsymbol{a}^{T} \boldsymbol{c}(\omega)+j \boldsymbol{a}^{T} \boldsymbol{s}(\omega)}{D_{0}(\omega, \boldsymbol{b})+j D_{1}(\omega, \boldsymbol{b})}
\end{aligned}
$$

where $D_{0}(\omega, \boldsymbol{b})$ and $D_{1}(\omega, \boldsymbol{b})$ are polynomials of $\boldsymbol{b}$. This leads (4b) to

$$
\delta D(\omega, \boldsymbol{b}) \geq P_{0}(\omega, \boldsymbol{a}, \boldsymbol{b})+P_{1}(\omega, \boldsymbol{a}, \boldsymbol{b}) \quad \omega \in \Omega
$$

where

$$
\begin{aligned}
& D(\omega, \boldsymbol{b})=D_{0}^{2}(\omega, \boldsymbol{b})+D_{1}^{2}(\omega, \boldsymbol{b}) \\
& P_{0}(\omega, \boldsymbol{a}, \boldsymbol{b})= \\
& W(\omega)\left[\boldsymbol{a}^{T} \boldsymbol{c}(\omega)-H_{d r}(\omega) D_{0}(\omega, \boldsymbol{b})-H_{d i}(\omega) D_{1}(\omega, \boldsymbol{b})\right]^{2} \\
& P_{1}(\omega, \boldsymbol{a}, \boldsymbol{b})= \\
& W(\omega)\left[\boldsymbol{a}^{T} \boldsymbol{s}(\omega)+H_{d r}(\omega) D_{1}(\omega, \boldsymbol{b})-H_{d i}(\omega) D_{0}(\omega, \boldsymbol{b})\right]^{2}
\end{aligned}
$$

If we define $\boldsymbol{x}=\left[\begin{array}{lll}\delta & \boldsymbol{a}^{T} & \boldsymbol{b}^{T}\end{array}\right]$ and $f(\omega, \boldsymbol{x})=\delta D(\omega, \boldsymbol{b})-$ $P_{0}(\omega, \boldsymbol{a}, \boldsymbol{b})-P_{1}(\omega, \boldsymbol{a}, \boldsymbol{b})$, then, for fixed $\omega, f(\omega, \boldsymbol{x})$ is a 3rdorder polynomial of $x$ and the constraint in (4b) becomes

$$
f(\omega, \boldsymbol{x}) \geq 0 \quad \omega \in \Omega
$$

Further notice that the stability constraint in (4c) is equivalent to [1]

$$
\begin{aligned}
1-\varepsilon-b_{j 0} & \geq 0 \\
1-\varepsilon+b_{j 0}-b_{j 1} & \geq 0 \\
1-\varepsilon+b_{j 0}+b_{j 1} & \geq 0
\end{aligned}
$$

where $1 \leq j \leq k$ and $\varepsilon$ is a small positive number to ensure a nonzero stability margin. Now if we impose constraint (5) only on a sufficiently dense set of grids $\Omega_{K}=\left\{\omega_{1}, \omega_{2}, \ldots, \omega_{K}\right\} \subseteq \Omega$, then the problem in (4) is reduced to

$$
\begin{aligned}
\operatorname{minimize} & \boldsymbol{e}_{1}^{T} \boldsymbol{x} \\
\text { subject to: } & f\left(\omega_{i}, \boldsymbol{x}\right) \geq 0 \quad \omega_{i} \in \Omega_{K} \\
& 1-\varepsilon-b_{j 0} \geq 0 \quad j=1, \ldots, k \\
& 1-\varepsilon+b_{j 0}-b_{j 1} \geq 0 \quad j=1, \ldots, k \\
& 1-\varepsilon+b_{j 0}+b_{j 1} \geq 0 \quad j=1, \ldots, k
\end{aligned}
$$

with $e_{1}=\left[\begin{array}{llll}1 & 0 & \cdots & 0\end{array}\right]^{T}$, which fits into the general POP formulation in (2).

\section{DESIGN OF FIR FRM FILTERS}

We refer the reader to Fig. 1 for a basic FIR FRM filter structure [4] which involves a linear-phase prototype filter $H_{a}(z)$ up-sampled by $M$, a pair of linear-phase masking filters $\left\{H_{m a}(z), H_{m c}(z)\right\}$, and a delay line that, together with the prototype filter, helps form a linear-phase complementory pair $\left\{H_{a}, H_{c}\right\}[4]$.

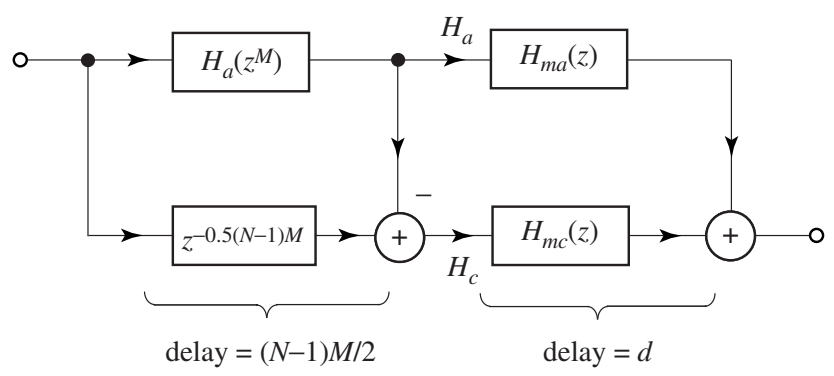

Fig. 1. Basic FRM filter structure.

Let the transfer functions in the FRM filter be given by

$$
H_{z}(z)=\sum_{k=0}^{N-1} h_{k} z^{-k}, H_{m a}(z)=\sum_{k=0}^{N_{a}-1} h_{k}^{(a)} z^{-k}
$$

and

$$
H_{m c}(z)=\sum_{k=0}^{N_{c}-1} h_{k}^{(c)} z^{-k}
$$

and for the sake of notation simplicity assume that $N, N_{a}$ and $N_{c}$ are odd integers, then it is known [14] that the zero-phase frequency response of the FRM filter is given by

$$
H(\omega, \hat{\boldsymbol{x}})=\left[\boldsymbol{a}^{T} \boldsymbol{c}(\omega)\right]\left[\boldsymbol{a}_{a}^{T} \boldsymbol{c}_{a}(\omega)-\boldsymbol{a}_{c}^{T} \boldsymbol{c}_{c}(\omega)\right]+\boldsymbol{a}_{c}^{T} \boldsymbol{c}_{c}(\omega)
$$

where

$$
\begin{aligned}
\boldsymbol{a} & =\left[\begin{array}{llll}
h_{(N-1) / 2} & 0.5 h_{(N+1) / 2} & \cdots & 0.5 h_{N-1}
\end{array}\right]^{T} \\
\boldsymbol{c}(\omega) & =\left[\begin{array}{llll}
1 & \cos M \omega & \cdots & \cos [(N-1) M \omega / 2]
\end{array}\right]^{T} \\
\boldsymbol{a}_{a} & =\left[\begin{array}{llll}
h_{\left(N_{a}-1\right) / 2}^{(a)} & 0.5 h_{\left(N_{a}+1\right) / 2}^{(a)} & \cdots & 0.5 h_{N_{a}-1}^{(a)}
\end{array}\right]^{T} \\
\boldsymbol{c}_{a}(\omega) & =\left[\begin{array}{llll}
1 & \cos \omega & \cdots & \cos \left[\left(N_{a}-1\right) \omega / 2\right.
\end{array}\right]^{T} \\
\boldsymbol{a}_{c} & =\left[\begin{array}{llll}
h_{\left(N_{c}-1\right) / 2}^{(c)} & 0.5 h_{\left(N_{c}+1\right) / 2}^{(c)} & \cdots & 0.5 h_{N_{c}-1}^{(c)}
\end{array}\right]^{T} \\
\boldsymbol{c}_{c}(\omega) & =\left[\begin{array}{llll}
1 & \cos \omega & \cdots & \cos \left[\left(N_{c}-1\right) \omega / 2\right.
\end{array}\right]^{T}
\end{aligned}
$$

and

$$
\hat{\boldsymbol{x}}=\left[\begin{array}{c}
\boldsymbol{a} \\
\boldsymbol{a}_{a} \\
\boldsymbol{a}_{c}
\end{array}\right]
$$

The weighted minimax design of the FRM filter is formulated as

$$
\underset{\boldsymbol{x}}{\operatorname{minimize}} \underset{\omega \in \Omega}{\operatorname{maximize}} W(\omega)\left|H(\omega, \hat{\boldsymbol{x}})-H_{d}(\omega)\right|
$$


where $H_{d}(\omega)$ is the zero-phase desired frequency response. By placing an upper bound for $W(\omega)\left|H(\omega)-H_{d}(\omega)\right|$ on a dense frequencies $\Omega_{K}=\left\{\omega_{1}, \ldots, \omega_{K}\right\} \subset \Omega$, the problem in (8) can be reformulated as

$$
\begin{aligned}
\operatorname{minimize} & \delta \\
\text { subject to: } & W\left(\omega_{i}\right)\left|H\left(\omega_{i}, \hat{\boldsymbol{x}}\right)-H_{d}\left(\omega_{i}\right)\right| \leq \delta
\end{aligned}
$$

for $\omega_{i} \in \Omega_{K}$, where the constraints in (9b) are equivalent to

$$
\begin{aligned}
& P_{0}\left(\omega_{i}, \boldsymbol{x}\right)=\delta-W\left(\omega_{i}\right)\left[H\left(\omega_{i}, \hat{\boldsymbol{x}}\right)-H_{d}\left(\omega_{i}\right)\right] \geq 0 \\
& P_{1}\left(\omega_{i}, \boldsymbol{x}\right)=\delta+W\left(\omega_{i}\right)\left[H\left(\omega_{i}, \hat{\boldsymbol{x}}\right)-H_{d}\left(\omega_{i}\right)\right] \geq 0
\end{aligned}
$$

where $\omega_{i} \in \Omega_{K}$ and $\boldsymbol{x}=\left[\begin{array}{ll}\delta & \hat{\boldsymbol{x}}^{T}\end{array}\right]^{T}$. Thus the problem in (9) can be stated as

$$
\begin{array}{cll}
\operatorname{minimize} & \boldsymbol{e}_{1}^{T} \boldsymbol{x} \\
\text { subject to: } & P_{0}\left(\omega_{i}, \boldsymbol{x}\right) \geq 0 & \\
& P_{1}\left(\omega_{i}, \boldsymbol{x}\right) \geq 0 & \omega_{i} \in \Omega_{K} \in \Omega_{K}
\end{array}
$$

where $P_{0}\left(\omega_{i}, \boldsymbol{x}\right)$ and $P_{1}\left(\omega_{i}, \boldsymbol{x}\right)$, according to (7) and (10), are non-convex second-order polynomials. Therefore (10) is a POP.

For the design of multistage FRM filters, the problem can be formulated in a similar way as POP with the order of $P_{0}\left(\omega_{i}, \boldsymbol{x}\right)$ and $P_{1}\left(\omega_{i}, \boldsymbol{x}\right)$ higher than two (see, e.g., Eq. (18) in [14]).

\section{Design of FIR Filters With PS2 CoefFicients}

It follows from [6][15] that the weighted least squares design of FIR digital filters with SP2 coefficients can be formulated as $\{-1,1\}$-optimization of a convex quadratic function.

$$
\begin{aligned}
\operatorname{minimize} & \boldsymbol{x}^{T} \boldsymbol{Q} \boldsymbol{x}+\boldsymbol{q}^{T} \boldsymbol{x} \\
\text { subject to: } & x_{k} \in\{-1,1\}, \quad k=1, \ldots, n
\end{aligned}
$$

The constraints in (11b) are equivalent to $x_{k}^{2}=1$ for $k=$ $1, \ldots, n$ which are in turn equivalent to $x_{k}^{2}-1 \geq 0$ and $x_{k}^{2}-1 \leq 0$ for $k=1, \ldots, n$. Hence the design problem is reduced to

$$
\begin{array}{rll}
\operatorname{minimize} & \boldsymbol{x}^{T} \boldsymbol{Q} \boldsymbol{x}+\boldsymbol{q}^{T} \boldsymbol{x} & \\
\text { subject to: } & x_{k}^{2}-1 \geq 0 & k=1, \ldots, n \\
& 1-x_{k}^{2} \geq 0 \quad k=1, \ldots, n
\end{array}
$$

which is obviously a POP.

\section{AN EXAMPLE}

Several MATLAB-compatible freeware for POPs are now available. These include SparsePOP [13] and GloptiPoly [16]. In what follows we present a simple design example of designing a 2nd-order stable IIR filter to approximate a lowpass frequency response with constant passband group delay, by using SparsePOP [13]. The transfer function in question assumes the form

$$
H(z)=\frac{a_{0}+a_{1} z+a_{2} z^{2}}{b_{0}+b_{1} z+z^{2}}
$$

The normalized passband and stopband edges were set to $\omega_{p}=$ $0.3 \pi$ and $\omega_{a}=0.7 \pi$ and passband group delay was set to $\tau=$ 1.2 . Both the passband and stopband were partitioned by equally spaced grids of density $0.025 \pi$. In this way, the POP has a total of $K=263$ rd-order constraints of type (6b) plus additional 3 linear constraints of type (6c)-(6e) for stability with $\varepsilon$ set to 0.01 . A piecewise constant weighting function $W(\omega)$, which was set to 1 in the passband and 0.5 in the stopband, was used. It took SparsePOP 20.42 seconds of CPU time on a $3.4 \mathrm{GHz}$ Pentium 4 $\mathrm{PC}$ to converge to the optimized coefficients

$$
\begin{aligned}
& a_{0}=0.20677997 \\
& a_{1}=0.32110153 \\
& a_{2}=0.21218703 \\
& b_{0}=0.31209347 \\
& b_{1}=-0.55720129
\end{aligned}
$$

The two poles are $0.2786 \pm j 0.4842$ whose modulus is 0.5587 . The maximum amplitude deviation in the passband was found to be 0.0472 and the minimum stopband attenuation was $25.6210 \mathrm{~dB}$. The amplitude response and passband group delay are depicted in Fig. $2 \mathrm{a}$ and $2 \mathrm{~b}$, respectively.

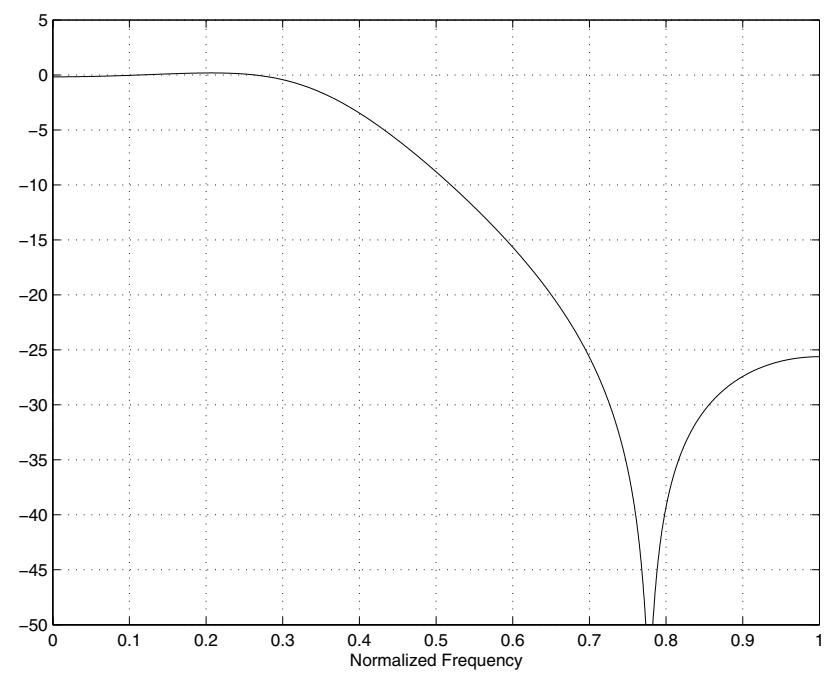

Fig. 2a. Amplitude response of the second-order IIR filter.

For the given design specifications, the filter obtained is believed to offer a nearly global solution in the sense that any design satisfying the same specifications and claiming to be a better design shall differ from the present design insignificantly.

\section{CONCLUding REMARKS}

A new design framework for digital filters based on polynomial optimization has been introduced. A striking feature of this design methodology is that it promises to generate global solutions for a variety of filter design problems. In this paper we have presented POP formulations for stable IIR filters, FIR FRM filters, and filters with discrete coefficients. There are however many other problems concerning multidimensional filters [17], filter banks [18], and filters with variable coefficients whose globally optimal designs can also be formulated and solved in the POP setting. Technical details for some of these solution methods will be made available in our forthcoming publications. 


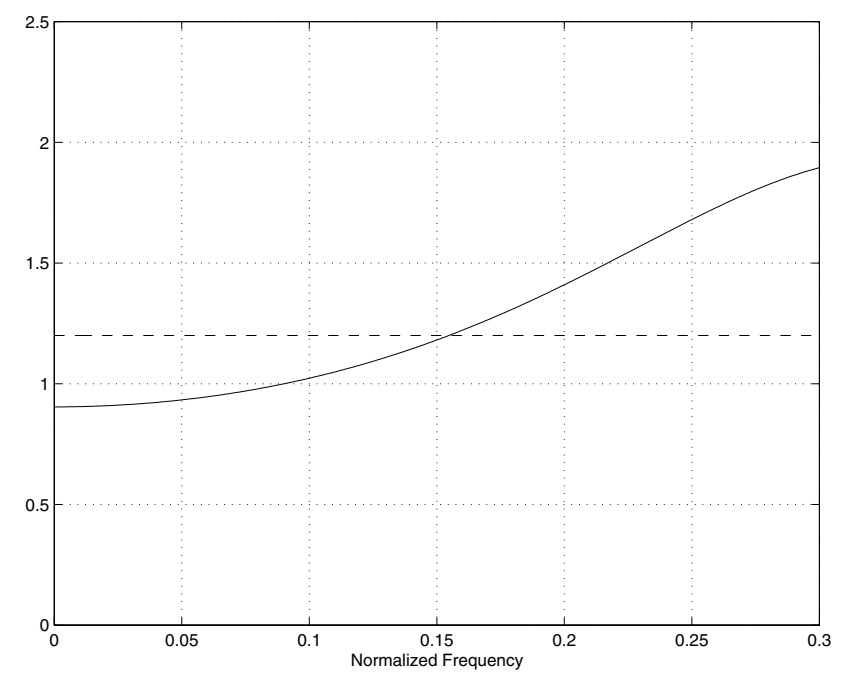

Fig. 2b. Passband group delay of the second order IIR filter.

\section{ACKNOWLEDGEMENT}

The author is grateful to the Natural Sciences and Engineering Research Council of Canada for supporting this work.

\section{REFERENCES}

[1] A. Antoniou, Digital Signal Processing, McGraw-Hill, 2006.

[2] S. K. Mitra, Digital Signal Processing, 3rd ed., McGraw-Hill, 2006.

[3] B. Gold and C. M. Radar, Digital Processing of Signals, McGraw-Hill, 1969

[4] Y. C. Lim, "Frequency-response masking approach for the synthesis of sharp linear phase digital filters," IEEE Trans. Circuits Syst., vol. 33, pp. 357-364, April 1986.

[5] Y. C. Lim and S. P. Parker, "FIR filter design over a discrete power-of-two coefficient space," IEEE Trans. ASSP, vol. 31, pp. 538-591, April 1983.

[6] W.-S. Lu, "Design of FIR filters with discrete coefficients: A semidefinite relaxation approach," ISCAS 2001, vo2, pp. 297-3300, Sydney, Australia, May 2001.

[7] N. Z. Shor, "Quadratic optimization problems," Soviet J. Comput. Systems Sci., vol. 25, pp. 1-11, 1987.

[8] Y. Nesterov, "Squared functional systems and optimization problems," in High Performance Opimization, H. Frenk, K. Roos, T. Terlaky, and S. Zhang eds., Kluwer, Dordrecht 2000.

[9] N. Z. Shor, Nondifferentiable Optimization and Polynomial Problems, Kluwer, Dordrecht, 1998.

[10] J. B. Lasserre, "Global optimization with polynomials and the problem of moments," SIAM J. Optimization, vol. 11, no. 3, pp. 796-817, 2001.

[11] H. Waki, S. Kim, M. Kojima, and M. Muramatsu, "Sum of squares and semidefinite programming relaxations for polynomial optimization problem with structured sparsity," to appear in SIAM J. Optimization.

[12] J. B. Lasserre, "An explicit equivalent positive semidefinite program for nonlinear D-1 programs," SIAM J. Optimization, vol. 12, no. 3, pp. 759-769, 2002.

[13] H. Waki, S. Kim, M. Kojima, and M. Muramatsu, "Sparse POP: A sparse semidefinite programming relaxation of polynomial optimization problems," Research Report, Tokyo Institute of Technology, March 2005.

[14] W.-S. Lu and T. Hinamoto, "Optimal design of frequency-resonse-masking filters using semidefinite programming," IEEE Trans. Signal Circuits Syst., I., vol. 50, pp. 557-568, April 2003.

[15] W.-S. Lu, "Design of FIR digital filters with discrete coefficients via sphere relaxation," ISCAS 2006, May 2006.

[16] D. Henrion and J. B. Lasserre, "GloptiPoly: Global optimization over polynomials with Matlab and SeDumi," LAAS-CNRS Report No. 02057, Toulouse, France 2002.

[17] W.-S. Lu and A. Antoniou, Two-Dimensional Digital Filters, Marcel Dekker, New York, 1992.

[18] P. P. Vaidyanathan, Multirate Systems and Filter Banks, Prentice Hall, 1993. 